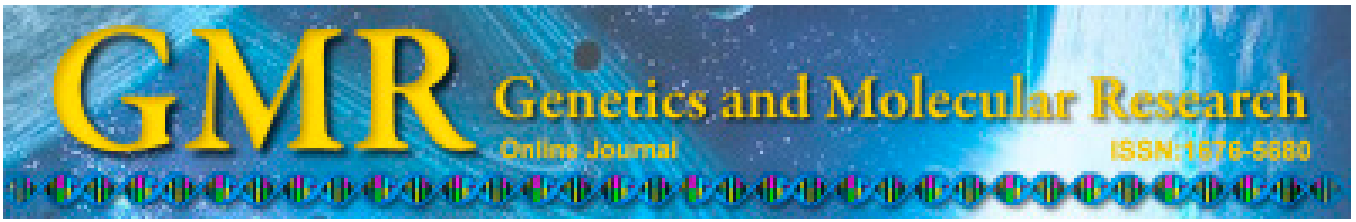

\title{
Development of RAPD-SCAR markers for different Ganoderma species authentication by improved RAPD amplification and molecular cloning
}

\author{
J.J. Fu' ${ }^{1,2}$, M.A. Khan ${ }^{1}$, Z.Q. Mei ${ }^{1}$, M. Tania ${ }^{1}$, L.Q. Yang ${ }^{1}$ and J.L. Cheng ${ }^{1}$ \\ ${ }^{1}$ Research Center for Preclinical Medicine, Sichuan Medical University, \\ Luzhou, Sichuan Province, China \\ ${ }^{2}$ Forensic Center, Sichuan Medical University, Luzhou, Sichuan, China \\ Corresponding author: J.J. Fu \\ E-mail: fujunjiang@hotmail.com
}

Genet. Mol. Res. 14 (2): 5667-5676 (2015)

Received August 28, 2014

Accepted January 19, 2015

Published May 25, 2015

DOI http://dx.doi.org/10.4238/2015.May.25.19

\begin{abstract}
The sequence-characterized amplified region (SCAR) is a valuable molecular technique for the genetic identification of any species. This method is mainly derived from the molecular cloning of the amplified DNA fragments achieved from the random amplified polymorphic DNA (RAPD). In this study, we collected DNA from 10 species of Ganoderma mushroom and amplified the DNA using an improved RAPD technique. The amplified fragments were then cloned into a T-vector, and positive clones were screened, indentified, and sequenced for the development of SCAR markers. After designing PCR primers and optimizing PCR conditions, 4 SCAR markers, named LZ14, LZ2-2, LZ8-2, and LZ9-15, were developed, which were specific to Ganoderma gibbosum (LZ1-4 and LZ8-2), Ganoderma sinense (LZ2-2 and LZ8-2), Ganoderma tropicum (LZ8-2), and Ganoderma lucidum HG (LZ9-15). These 4 novel SCAR markers were deposited into GenBank with the accession Nos. KM391935, KM391936, KM391937,
\end{abstract}


and KM391938, respectively. Thus, in this study we developed specific SCAR markers for the identification and authentication of different Ganoderma species.

Key words: Genetic identification; Random amplified polymorphic DNA; Ganoderma species; Sequence-characterized amplified region

\section{INTRODUCTION}

In East Asian countries, particularly China and Japan, mushrooms of Ganoderma species have been cultivated and used as traditional medicines for thousands of years. They are commonly known as 'Reishi' in Japan or as 'língzhỉ' in China. Ganoderma mushrooms are popular because of their therapeutic potential against several life-threatening diseases. Among different species, Ganoderma lucidum is the most popular and well explored for its beneficial health activities (Paterson, 2006; Sanodiya et al., 2009; Mahajna et al., 2009; Mei et al., 2014a). Other species have gained less attention; however, they also have significant medicinal values. For example, Ganoderma sinense has immunomodulatory, anticancer, and antiviral activities (Liu et al., 2009; Sato et al., 2009; Yue et al., 2013), while Ganoderma tropicum has acetylcholinesterase inhibitory activity and neuro-beneficial activity (Hu et al., 2013). Ganoderma gibbosum is also used as a medicinal fungus in China (Chen et al., 2010). These Ganoderma mushrooms possess numerous variations in the size, shape, and color, and it is often difficult to identify and morphologically distinguish them. Thus, genetic characterization and identification are important for Ganoderma mushrooms.

Random amplified polymorphic DNA (RAPD), inter-simple sequence repeat, simple sequence repeat, and amplified fragment length polymorphism analyses are the major molecular marker technologies currently used for the genetic characterization and identification of an organism (Williams et al., 1990; Agarwal et al., 2008; Fu et al., 2013; Noormohammadi et al., 2013; Mei et al., 2014a,b). The sequence characterized amplified region (SCAR) marker is another molecular marker, which is more stable and is generally derived from RAPD or intersimple sequence repeat (Dnyaneshwar et al., 2006; Su et al., 2007; Li et al., 2010; Kumla et al., 2012; Rajesh et al., 2013). When SCAR with RAPD are combined, molecular analysis is simplified, in which PCR primers are designed from the sequence of the RAPD amplicon to develop SCAR markers (Kumla et al., 2012; Rajesh et al., 2013).

We previously used an improved RAPD technique for the genetic characterization of different Ganoderma species (Mei et al., 21014a). In this study, we developed 4 SCAR markers after the molecular cloning of RAPD fragments obtained from the DNA materials of different Ganoderma species. These markers were found to be specific to certain Ganoderma species.

\section{MATERIAL AND METHODS}

\section{Extraction of DNA from Ganoderma species}

DNA was extracted from different samples of Ganoderma species (Table 1) and other samples by using standard methods. DNA samples were then diluted to a final concentration of $10 \mathrm{ng} / \mu \mathrm{L}$ and stored at $-20^{\circ} \mathrm{C}$ until use (Fu et al., 2013). 


\begin{tabular}{|c|c|c|c|}
\hline No. & Accession name & Sources of Ganoderma & Deposit No. \\
\hline 1 & Ganoderma gibbosum (Blumii et Nees) Patouillard & Guangdong Culture Collection Center & GIM5.6 \\
\hline 2 & Ganoderma tropicum (Jungh.) Bres. & Guangdong Culture Collection Center & GIM5.289 \\
\hline 3 & Ganoderma applanatum (Pers.ex Wullr) Pat & Guangdong Culture Collection Center & GIM5.282 \\
\hline 4 & Ganoderma australe (Fr.) Pat & Guangdong Culture Collection Center & GIM5.288 \\
\hline 5 & Ganoderma sinense & Inst. Microbiology of Chinese Academy of Sciences & CGMCC5.0069 \\
\hline 6 & Ganoderma lucidum $\mathrm{HG}$ & Inst. Edible Fungi of Fujian academy of Agricultural Sciences & ACCC51329 \\
\hline 7 & Ganoderma lucidum (Curtis) P. Karst & Inst. Edible Fungi of Fujian Academy of Agricultural Sciences & CFCC85862 \\
\hline 8 & Ganoderma lucidium & Inst. Microbiology of Chinese Academy of Sciences & CGMCC5.0026 \\
\hline 9 & Ganoderma neojaponicum Imazeki & Beijing Agricultural University & CFCC87599 \\
\hline 10 & Ganoderma lucidium (Leysser Fr.) Karst. & Inst. Wensheng Edible Fungi in Shantou & GIM5.250SL \\
\hline
\end{tabular}

\section{Amplification and recovery of improved RAPD fragments}

The improved RAPDs were amplified by polymerase chain reaction (PCR) using the random primers SBC-M7 and SBC-I1 DNA from 10 Ganoderma species (Table 1). The 15 $\mu \mathrm{L}$ PCR mixture consisted of $7.5 \mu \mathrm{L} 2 \mathrm{X}$ Taq PCR MasterMix, $1.5 \mu \mathrm{L} 2.5 \mu \mathrm{M}$ primer, $1.5 \mu \mathrm{L}$ genomic DNA, and $\mathrm{ddH}_{2} \mathrm{O}$. Amplification reactions were performed using the machine Veriti ${ }^{\circledR}$ 96-Well Thermal Cycler" (Applied Biosystems, Foster City, CA, USA), with the following steps: initial denaturation at $95^{\circ} \mathrm{C}$ for $90 \mathrm{~s}, 40$ cycles of denaturation at $94^{\circ} \mathrm{C}$ for $40 \mathrm{~s}$, annealing at $36^{\circ} \mathrm{C}$ for $60 \mathrm{~s}$, with the annealing to extension temperature increasing at $0.125^{\circ} \mathrm{C} / \mathrm{s}$ ( $5 \%$ ramp rate), extension at $72^{\circ} \mathrm{C}$ for $90 \mathrm{~s}$, and a final extension step at $72^{\circ} \mathrm{C}$ for $5 \mathrm{~min}$. PCR products were separated on a $1.5 \%$ agarose gel by electrophoresis for $40 \mathrm{~min}$. The bright bands were excised from the agarose gel, purified using the TIANgel Mini Purification Kit (DP209, Tiangen Biotech, Beijing, China) according to the manufacturer protocol.

\section{Cloning, screening, and sequencing of DNA fragments}

The purified DNA fragments were ligated into the pGM-T vector (No. VT202, Tiangen) and transformed into DH5 $\alpha$ Escherichia coli competent cells. The recombinant clones were selected on Luria Bertani (LB) agar plates containing $100 \mu \mathrm{g} / \mu \mathrm{L}$ ampicillin, $40 \mathrm{mg}$ 5-bromo-4-chloro-3-indoyl- $\beta$-D-galactopyranoside, and $160 \mu \mathrm{g}$ isopropyl $\beta$-D-1-thiogalactopyranoside. The white colonies were screened out by blue/white screening. The presence of the correct insert was verified by PCR using the T7/SP6 primer pairs (Yang et al., 2013), located in the pGM-T vector near the ligation ends, and EcoRI digestion (Fu, 2012). The cloned DNA fragments were then sequenced using the Sanger method.

\section{Homological analysis and SCAR primer design}

The homology of sequenced DNA was searched and analyzed using the online program BLAST (http://www.ncbi.nlm.nih.gov/BLAST/) in different species. The nucleotide sequence of each cloned RAPD fragment was used to design pairs of SCAR primers using Primer 3 (http:// bioinfo.ut.ee/primer3-0.4.0/primer3/). The sequences of each primer are listed in Table 2.

\section{Development SCAR markers and SCAR analysis}

To develop SCAR markers, PCR amplification was performed. The $10 \mu \mathrm{L}$ PCR system 
Table 2. Sequences of SCAR primers, PCR condition and product size.

\begin{tabular}{lllllcc}
\hline SCAR & 5'-primer & Sequence $\left(5^{\prime}-3^{\prime}\right)$ & 3'-primer & Sequence $\left(5^{\prime}-3^{\prime}\right)$ & Size $(\mathrm{bp})$ & Tm $\left({ }^{\circ} \mathrm{C}\right)$ \\
\hline LZ1-4 & LZ1-4L & GTGTTTCTGGCATGCACACC & LZ1-4R & ACACAGTACTTCACCGACGG & 211 & 62 \\
LZ2-2 & LZ2-2L & TGAGGATTGGAAACGGGGTG & LZ2-2R & CTCTGGTGTTTGGATTGCGC & 272 & 62 \\
LZ8-2 & LZ8-2L & AACCGCCAAGACACTGTAGG & LZ8-2R & CTCTCATCGGGTTCACTCGG & 291 & 60 \\
LZ9-15 & LZ9-15L & ACCACCTACCTGCTCCTCTT & LZ9-15R & TCCTTCCGGCAGTGGTAGTA & 262 & 60 \\
\hline
\end{tabular}

included: $5 \mu \mathrm{L} 2 \mathrm{X}$ Taq PCR MasterMix, $1 \mu \mathrm{L} 2.5 \mu \mathrm{M}$ of each SCAR primer, $1 \mu \mathrm{L} 10 \mathrm{ng}$ genomic DNA, and $3 \mu \mathrm{L}$ ddH ${ }_{2} \mathrm{O}$. PCR was performed by using the Veriti ${ }^{\circledR} 96-$ Well Thermal Cycler (Applied Biosystems) with an initial pre-denaturation for $90 \mathrm{~s}$ at $95^{\circ} \mathrm{C}$, followed by 35 cycles of denaturation at $94^{\circ} \mathrm{C}$ for $40 \mathrm{~s}$, annealing at temperatures $60^{\circ}$ or $62^{\circ} \mathrm{C}$ for $30 \mathrm{~s}$, and extension at $72^{\circ} \mathrm{C}$ for $40 \mathrm{~s}$. The final extension step was performed at $72^{\circ} \mathrm{C}$ for $5 \mathrm{~min}$. Sequences of the 4 pairs of SCAR primers, amplified length, and PCR conditions are listed in Table 2. To identify differences between the varieties of Ganoderma sp and other species of medicinal plants, SCAR analysis was performed using 23 DNA samples as templates, including 10 samples of Ganoderma sp, which were described previously (Mei et al., 2014a), 2 samples of Dimocarpus longan collected from Fujian and Hainan (Yang et al., 2013), 2 samples of Lonicera japonica collected from Guangdong and Hubei (Fu et al., 2013), 1 Gardenia jasminoides, 1 Litchi chinensis from Guangdong, 1 Dimocarpus confinis from Guangxi, 1 Canarium album from Luzhou City, Gastrodia elata collected from Liangshan City in Sichuan Province, 1 Penthorum. sedoides, Penthorum chinense collected from Gulin County in Sichuan Province, and Violoa philippica (Yang et al., 2013), and 1 Angelica sinensis from Sichuan Province. The amplified PCR products were separated by electrophoresis on a $1.0 \%$ agarose gel in $1 \mathrm{X}$ TAE buffer at $120 \mathrm{~V}$ for $40 \mathrm{~min}$. Gels were visualized by $0.5 \mu \mathrm{g} / \mathrm{mL}$ ethidium bromide staining, and the images were documented using the ChemiDoc XRS (Bio-Rad, Hercules, CA, USA).

\section{RESULTS}

\section{Recovery of RAPD fragments}

Two RAPD primers, SBC-M7 and SBC-I1, were used to improve RAPD amplification from DNA samples of Ganoderma species (Mei et al., 2014a). The results are shown in Figure 1A; the blue arrows indicate bands that were cut from the agarose gel and labeled with L1, L2, L8, and L9, respectively. DNA from the agarose gel was purified using the TIANgel Mini Purification Kit and eluted with $20 \mu \mathrm{LddH}_{2} \mathrm{O}$. To check DNA quality and measure the quantity for ligation, $2 \mu \mathrm{L}$ purified PCR products and $0.5 \mu \mathrm{L}$ T-vector was added to each well of an agarose gel. Figure 1B shows the DNA quality and quantity.

\section{Cloning of RAPD fragments}

Based on DNA quality, an appropriate amount of PCR product and T-vector were ligated together and screened using the blue/white method. Positive clones were then identified by PCR amplification using SP6/T7 primers (Figure 2). The plasmids from positive clones in Figure 2 in red were further extracted and cut by EcoRI enzyme digestion (Figure 3). In lane 2 of Figure 3, clone LZ1-4, which showed a 550-base pair (bp) DNA fragment, was sequenced. In lane 4 of Figure 3, clone LZ2-2, which showed a 600-700-bp inserted DNA fragment, was 
sequenced. In lane 6 of Figure 3, clone LZ8-2, which showed a 550-650-bp inserted DNA fragment, was sequenced. In lane 8 of Figure 3, clone LZ9-15, which showed a 550-650-bp inserted DNA-fragment, was sequenced.

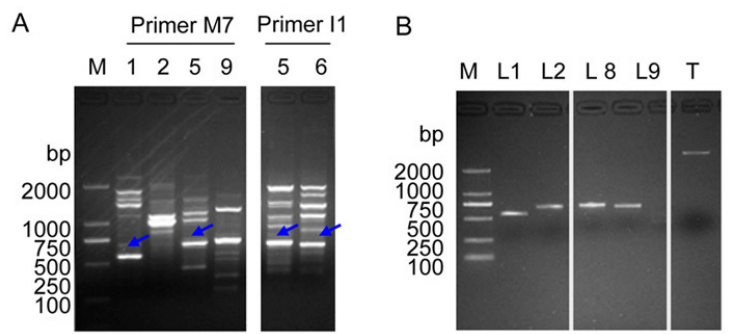

Figure 1. Amplification, quality and quantity checking of improved RAPD fragments. A. Improve RAPD amplification from DNA samples of Ganoderma species. Lanes 1, 2, 5, 6, and $9=$ different Ganoderma species samples listed in Table 1. The blue arrows indicate bands before cut. B. Quality and quantity checking of improved RAPD fragments purified from agarose. Lanes $L 1, L 2, L 8$, and $L 9$ indicate the PCR fragments LZ1, LZ2, LZ8, and LZ9, respectively. "T" is T vector. Lane M indicates the DNA molecular weight marker DL2000 with the fragment size of 2000, 1000, 750, 500, 250, $100 \mathrm{bp}$.
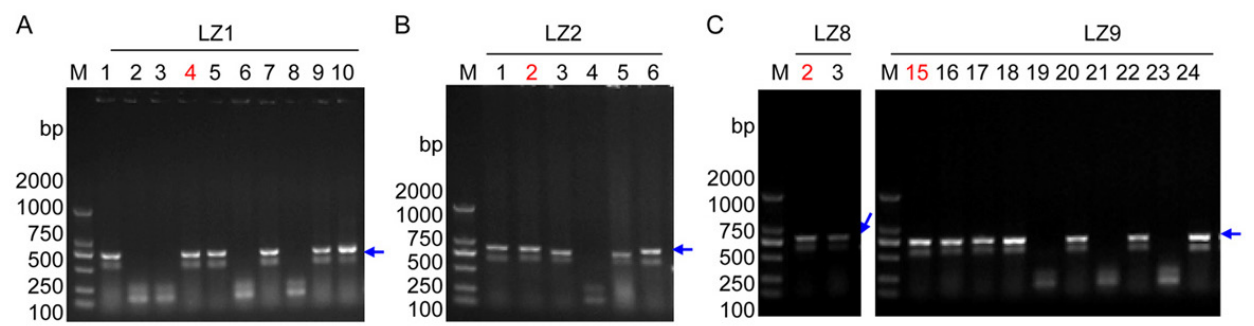

Figure 2. DNA cloning and identification of positive clones. A. Clone identification of RAPD fragment LZ1. Lanes 1-10 indicate different clones. B. Clone identification of RAPD fragment LZ2. Lanes 1-6 indicate different clones. C. Clone identification of RAPD fragment LZ8. Lanes 2-3 indicate different clones. Clone identification of RAPD fragment LZ9. Lanes 15-24 indicate different clones. The blue arrows indicate positive PCR products. Clones LZ14, LZ2-2, LZ8-2 and LZ9-15 in red colors were picked up to extract the plasmid DNA. The blue arrows indicate expected PCR bands in size of different clones. Lane $M$ indicates the DNA molecular weight marker DL2000 with the fragment size of 2000, 1000, 750, 500, 250, $100 \mathrm{bp}$.

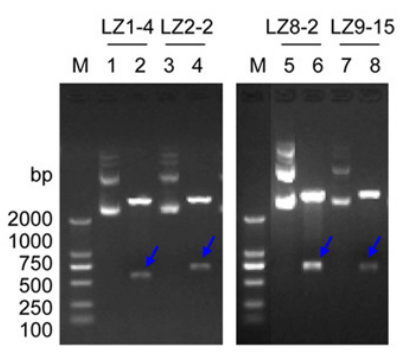

Figure 3. Identification of positive clones by plasmid DNA digestion. Lanes 1 and 2 indicate clone LZ1-4 plasmid DNA without or with EcoRI digestion. Lanes 3 and 4 indicate clone LZ2-2 plasmid DNA without or with EcoRI digestion. Lanes 5 and 6 indicate clone LZ8-2 plasmid DNA without or with EcoRI digestion. Lanes 7 and 8 indicate clone LZ915 plasmid DNA without or with EcoRI digestion. The blue arrows indicate expected inserts of RAPD DNA fragments. 


\section{Sequences and characterization of Ganoderma species-specific RAPD fragments}

Sequencing of the above 4 cloned RAPD fragments revealed that clone LZ1-4 consisted of 555 nucleotides and was deposited into GenBank with accession No. KM391935 (Figure 4A), clone LZ2-2 consisted of 658 nucleotides and was deposited into GenBank with accession No. KM391936 (Figure 4B), clone LZ8-2 consisted of 598 nucleotides and was deposited into GenBank with accession No. KM391937 (Figure 4C), and clone LZ9-15 consisted of 567 nucleotides and was deposited into GenBank with accession No. KM391938 (Figure 4B). BLAST searches of the nucleotide sequences in GenBank showed that no cloned DNAs showed identity to other species (data not shown).

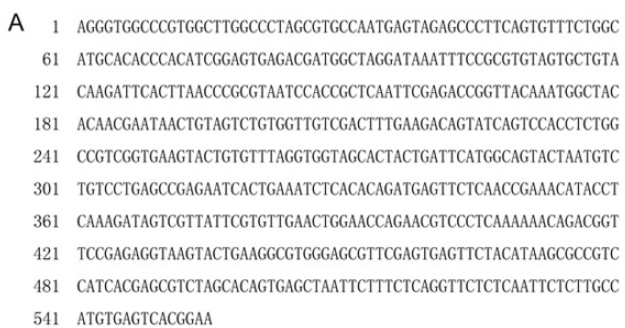

C

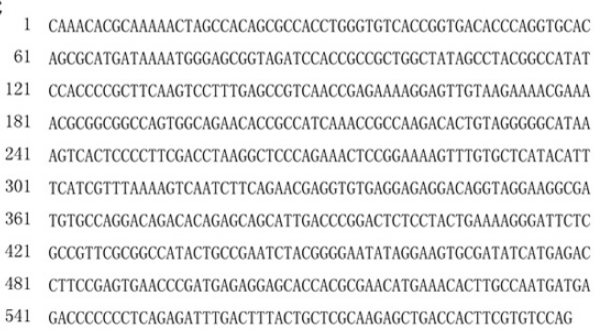

D

1 CATAGAGGACTTCATGCTCTGCAAAGGGGAGAACCGGGACCCGGCCCACTGTCTGAAGGA 61 GGGACGACGAGTGACGCGCTGGGCGCAGGACCTGTACGTGCCGGCCACCACCTACCTGCT 121 СCTCTTTCTCCGCCGTCGCTCACTGCCCACTCCCACACCGCACCCTCTTGGGAAACCGAC 181 TTACCCTGCTGTCGCCTGCCGCCTGTGAACAGGATCACAAAGCTTCGGGAAACTGCCTC 241 TCGGAGTTTGAGAAGCACTGGAACTGCTTGGAATATAACAACCAGGCATGTCTCATCCAC 301 TTCTTGTGCCTCCCGTTATGTTGATCCGACCCACCGGGCGAGTAGGAATACTACCACTGC 361 CGGAAGGACGAGCGGGTGCTGAACAAGTGGGTGTTCGAGAAGTTGGTGAGTTACCATTTT 421 TGTCGCGGTCATCGACTCGCCGCCGTTGCATATACTGTAAACTGACTGTACGACTCGGCA 481 TAGGGCCTCACGAAGACCATTCCGGGCTCGCCCGAAGGCAAAACGCCGATTCATGAGGTC 541 AAGAACCCCGTCTTCACTGGTGTCCAG

Figure 4. Cloned sequences information by Sanger-sequencing. A. Sequences of clone LZ1-4. B. Sequences of clone LZ2-2. C. Sequences of clone LZ8-2. D. Sequences of clone LZ9-15.

\section{Development of Ganoderma species-specific SCAR markers}

To generate stable diagnostic Ganoderma species-specific SCAR markers from RAPD markers, 4 pairs of primers (Table 2) were designed and synthesized based on cloned sequences. The designed SCAR primer pairs were then used to amplify genomic DNA from 23 of collected DNA samples to test amplification species-specificity. The results are shown in Figure 5, the SCAR maker LZ1-4 showed an expected size in a sample G. gibbosum (Blumii et Nees) Patouillard of 10 Ganoderma species (lane 1 of Figure 5A), without any amplification in other species tested; the SCAR maker LZ2-2 showed an expected size in a Ganoderma sinense sample of 10 Ganoderma species (in lane 5 of Figure 5B), without any amplification in other species tested; the SCAR marker LZ8-2 showed an expected size in a G. sinense sample of 10 Ganoderma species (lane 5 of Figure 5C) and 2 weak bands in the sample of G. gibbosum (Blumii et Nees) Patouillard (lane 1 of Figure 5C), and sample of G. tropicum (Jungh.) Bres (lane 2 of Figure 5C), without any amplification in other species tested; the SCAR maker 
LZ9-15 showed an expected size in an G. lucidum HG sample of 10 Ganoderma species (lane 6 of Figure 5D), without amplification in other species tested. These results indicate that these SCAR markers were sample-specific, not species-specific. The lack of this specific amplicon in the intra-species or other species indicates the efficacy of this marker for distinguishing the Ganoderma samples.



Figure 5. Development of RAPD-SCAR markers for LZ1-4, LZ2-2, LZ8-2 and LZ9-15. A. A SCAR marker LZ14. B. A SCAR marker LZ2-2. C. A SCAR marker LZ8-2. D. A SCAR marker LZ9-15. Lanes 1-10 indicate the different Ganoderma species samples listed in Table 1. Lanes 11-23 are DNA samples prescribed in Material and Methods. Lanes 11 and 12 are two samples of Dimocarpus longan; Lanes 13 and 14 are two samples of Lonicera japonica; lane 15 is one sample of Gardenia jasminoides; lane 16 is one sample of Litchi chinesis; lane 17 is one sample of Dimocarpus confinis; lane 18 is one sample of Canarium album; lane 19 is one sample of Gastrodia elata; lane 20 is one sample of Penthorum sedoides; lane 21 is one sample of Penthorum chinense; lane 22 is one sample of Violoa philippica; lane 22 is one sample of Angelica sinensis. The red arrows indicate expected PCR products in size. Lane $M$ indicates the DNA molecular weight marker DL2000.

\section{DISCUSSION}

Mushrooms in the Ganoderma genus are consumed as a source of natural medicine and are known for their health benefits and therapeutic activities. Some species of the Ganoderma mushrooms are more effective than others in terms of edibility and medicinal usage. Identification of specific Ganoderma species would be helpful for consumers, health professionals, and systemic mycobiologists. In this study, we developed SCAR molecular markers for the identification of 3 species of Ganoderma genus based on previous RAPD genetic char- 
acterization (Mei et al., 2014a).

Molecular marker technology, particularly the RAPD technique, and improved RAPD analysis has become a common and efficient tool for molecular analysis or genetic characterization of different species. A combination of SCAR and RAPD has significantly improved the stability and authenticity of the technique (Dnyaneshwar et al., 2006; Su et al., 2007; Li et al., 2010; Kumla et al., 2012; Rajesh et al., 2013; Fu et al., 2013; Noormohammadi et al., 2013; Mei et al., 2014a,b). Previous studies successfully developed RAPD markers for different species of animals, plants, and microbes (Lee et al., 2013; Yang et al., 2013, 2014; Dutta et al., 2014). However, no SCAR marker for the identification of different Ganoderma species has been reported. In this study, we developed 4 SCAR markers for the identification of different species, including G. gibbosum, G. sinense, G. tropicum, and G. lucidum HG.

The 555-nucleotide SCAR marker LZ1-4 was established to identify or authenticate G. gibbosum, which has been deposited into GenBank with accession No. KM391935, while LZ2-2 of 658 nucleotides has been deposited into GenBank with accession No. KM391936 specific to G. sinense. The LZ8-2 marker of 598 nucleotides was established to identify $G$. sinense; however, this marker also possesses a weaker level of specificity to G. gibbosum and G. tropicum. The GenBank accession No. for LZ8-2 is KM391937. Another SCAR marker, LZ9-15, was found to be specific to G. lucidum HG. This marker consists of 567 nucleotides, and has been deposited in to GenBank with the accession No. KM391938. Because BLAST searches of the nucleotide sequences in GenBank revealed no matches to these sequences, these novel markers can be used for the identification of these 4 species of Ganoderma mushroom. Typically, the different species of Ganoderma mushrooms vary in morphology and chemical constituents. G. lucidium (Leysser Fr.) Karst and G. lucidium HG are relatively well-known for their nutritional and medicinal importance compared to other species, such as G. gibbosum, G. tropicum, and G. sinense, while G. lucidum is bitter in taste compared to G. sinense. The variable medicinal importance may be because different Ganoderma species have different genetic characteristics and their genetic materials are highly polymorphic. The diverse range of genetic characteristics of Ganoderma mushrooms also influences their growth conditions. For example, G. lucidum HG is typically grown in Northern and North-Eastern China in cold environments, while G. tropicum is grown in tropical forests. A sequence-related amplified polymorphism analysis by Sun et al. (2006) revealed significant genetic variation between G. lucidum and G. sinense. Zhao et al. (2003) showed $80-100 \%$ polymorphism in genetic materials in different Ganoderma species, and Mei et al. (2014b) showed high levels of genetic distance between G. gibbosum, G. tropicum, G. sinense, and G. lucidum. This high genetic variability enabled the generation of species-specific SCAR markers. These molecular markers may have roles in ecological preservation, molecular identification, and authentication, as well as genetic characterization of these medicinal mushrooms.

\section{Conflicts of interest}

The authors declare no conflict of interest.

\section{ACKNOWLEDGMENTS}

Research supported partly by the Science and Technology Innovation Team of Colleges and Universities in Sichuan Province (\#13TD0032), the Applied Basic Research Program of 
Science and Technology Department of Sichuan Province (\#2014JC0121), and the Luzhou City Special Foundation (\#2013LZLY-J10).

\section{REFERENCES}

Agarwal M, Shrivastava N and Padh H (2008). Advances in molecular marker techniques and their applications in plant sciences. Plant Cell Rep. 27: 617-631.

Chen X, Zeng N and Lan J (2010). Cultural characteristics of mycelia of Ganoderma gibbosum. Zhongguo Zhong Yao Za Zhi. 35: 1939-1942.

Dnyaneshwar W, Preeti C, Kalpana J and Bhushan P (2006). Development and application of RAPD-SCAR marker for identification of Phyllanthus emblica LINN. Biol. Pharm. Bull. 29: 2313-2316.

Dutta S, Biswas S, Mukherjee K, Chakrabarty U, et al. (2014). Identification of RAPD-SCAR marker linked to white spot syndrome virus resistance in populations of giant black tiger shrimp, Penaeus monodon Fabricius. J. Fish Dis. 37: 471-480.

Fu J, Yang L, Khan MA and Mei Z (2013). Genetic characterization and authentication of Lonicera japonica Thunb. by using improved RAPD analysis. Mol. Biol. Rep. 40: 5993-5999.

Fu JJ (2012). Short protocols in medical molecular biology. China Medical Science Press, Beijing.

Kumla S, Doolgindachbaporn S, Sudmoon R and Sattayasai N (2012). Genetic variation, population structure and identification of yellow catfish, Mystus nemurus $(\mathrm{C} \& \mathrm{~V})$ in Thailand using RAPD, ISSR and SCAR marker. Mol. Biol. Rep. 39: 5201-5210.

Liu YW, Gao JL, Guan J, Qian ZM, et al. (2009). Evaluation of antiproliferative activities and action mechanisms of extracts from two species of Ganoderma on tumor cell lines. J. Agric. Food Chem. 57: 3087-3093.

Hu LL, Ma QY, Huang SZ, Guo ZK, et al. (2013). Three new lanostanoid triterpenes from the fruiting bodies of Ganoderma tropicum. J. Asian Nat. Prod. Res. 15: 357-362.

Lee DH, Lee SK, Lee SY and Lee JK (2013). Development of SCAR Markers for the Identification of Phytophthora katsurae Causing Chestnut Ink Disease in Korea. Mycobiology 41: 86-93.

Li SF, Tang SJ and Cai WQ (2010). RAPD-SCAR markers for genetically improved NEW GIFT Nile tilapia (Oreochromis niloticus niloticus L.) and their application in strain identification. Dongwuxue Yanjiu. 31: 147-153.

Mahajna J, Dotan N, Zaidman BZ, Petrova RD, et al. (2009). Pharmacological values of medicinal mushrooms for prostate cancer therapy: the case of Ganoderma lucidum. Nutr. Cancer 61: 16-26.

Mei Z, Yang L, Khan MA, Yang M, et al. (2014a). Genotyping of Ganoderma species by improved random amplified polymorphic DNA (RAPD) and inter-simple sequence repeat (ISSR) analysis. Biochem. Syst. Ecol. 56: 40-48.

Mei ZQ, Fu SY, Yu HQ, Yang LQ, et al. (2014b) Genetic characterization and authentication of Dimocarpus longan Lour. using an improved RAPD technique. Genet. Mol. Res. 13: 1447-1455.

Noormohammadi Z, Hasheminejad-Ahangarani FY, Sheidai M, Ghasemzadeh-Baraki AS, et al. (2013). Genetic diversity analysis in Opal cotton hybrids based on SSR, ISSR, and RAPD markers. Genet. Mol. Res. 12: 256-269.

Paterson RR (2006). Ganoderma - a therapeutic fungal biofactory. Phytochemistry 67: 1985-2001.

Rajesh MK, Jerard BA, Preethi P, Thomas RJ, et al. (2013). Development of a RAPD-derived SCAR marker associated with tall-type palm trait in coconut. Sci. Hort. 150: 312-316.

Sanodiya BS, Thakur GS, Baghel RK, Prasad GB, et al. (2009). Ganoderma lucidum: a potent pharmacological macrofungus. Curr. Pharm. Biotechnol. 10: 717-742.

Sato N, Zhang Q, Ma CM and Hattori M (2009). Anti-human immunodeficiency virus-1 protease activity of new lanostanetype triterpenoids from Ganoderma sinense. Chem. Pharm. Bull. 57: 1076-1080.

$\mathrm{Su}$ H, Wang L, Ge Y, Feng E, et al. (2007). Development of strain-specific SCAR markers for authentication of Ganoderma lucidum. World J. Microbiol. Biotechnol. 24: 1223-1226.

Sun SJ, Gao W, Lin SQ, Zhu J, et al. (2006). Analysis of genetic diversity in Ganoderma population with a novel molecular marker SRAP. Appl. Microbiol. Biotechnol. 72: 573-543.

Williams JG, Kubelik AR, Livak KJ, Rafalski JA, et al. (1990). DNA polymorphisms amplified by arbitrary primers are useful as genetic markers. Nucleic Acids Res. 18: 6531-6535.

Yang L, Fu S, Khan MA, Zeng W, et al. (2013). Molecular cloning and development of RAPD-SCAR markers for Dimocarpus longan variety authentication. SpringerPlus 2: 501.

Yang L, Khan MA, Mei Z, Yang M, et al. (2014). Development of RAPD-SCAR markers for Lonicera japonica Thunb. (Caprifolicaceae) variety authentication by improved RAPD and DNA cloning. Rev. Biol. Trop. 62: 1649-1657.

Yue GG, Chan BC, Han XQ, Cheng L, et al. (2013). Immunomodulatory activities of Ganoderma sinense polysaccharides 
in human immune cells. Nutr. Cancer 65: 765-774.

Zhao MW, Chen MJ, Wang N, Liang WQ, et al. (2003). Study on genetic relationship among some commercial strains of Ganoderma. J. Nanjing Agric. Univ. 26: 60-63. 